\title{
Clinicopathological correlation of an excised choroidal neovascular membrane in pseudotumour cerebri
}

\author{
Alessandro A Castellarin, Ilene K Sugino, Ma'an Nasir, Marco A Zarbin
}

\begin{abstract}
Aims/background-To correlate the histopathology of an excised choroidal neovascular membrane (CNV) with the clinical and angiographic findings in a 32-year-old woman with pseudotumour cerebri and a peripapillary CNV with subfoveal extension.

Methods-The patient's visual acuity was assessed by individuals experienced in low vision refraction and who were not members of the surgical team. The CNV was excised via a conventional three port vitrectomy with subretinal dissection. The excised tissue was studied with light and electron microscopy. Preoperative and serial postoperative fluorescein angiograms (FAs) and fundus photographs were obtained to study the dissection bed. Results-One week after surgery, the FA showed mottled subfoveal choriocapillaris perfusion. Three weeks after surgery, this area showed retinal pigment epithelium (RPE) atrophy clinically, and the FA showed choriocapillaris non-perfusion. Six months after surgery, the area of RPE atrophy and the corresponding area of choriocapillaris non-perfusion had expanded. Histologically, the excised CNV disclosed hyperplastic RPE, fibrovascular tissue, and no choriocapillaris. Fragments of RPE basement were present along the external edge of the specimen. The patient's visual acuity did not improve significantly after surgery.

Conclusions-Choriocapillaris nonperfusion can develop even in young patients following CNV excision. In this particular case, it is believed that choriocapillaris atrophy was caused by incomplete ingrowth of RPE into the dissection bed following RPE removal with CNV excision. As far as is known, this is the first report describing the results of surgery for CNV secondary to papilloedema associated with pseudotumour cerebri. (Br f Ophthalmol 1997;81:994-1000)
\end{abstract}

Correspondence to: Marco A Zarbin, $\mathrm{MD}, \mathrm{PhD}$, University of Medicine and Dentistry, New Jersey

Medical School, Department of Ophthalmology, 90 Bergen Street, 6th floor, Newark, NJ 07103-2499, USA.

Accepted for publication 25 June 1997

Choroidal neovascularisation is an uncommon sequela of papilloedema associated with pseudotumour cerebri. Jamison first described a case of unilateral peripapillary choroidal neovascularisation in a patient with bilateral papilloedema secondary to pseudotumour cerebri in $1978 .{ }^{1}$ Other cases have been reported by Troost et $a l,{ }^{2}$ Morris and Sanders, ${ }^{3}$ Morse et $a l^{4}{ }^{4}$ and Caballero-Presencia et al. ${ }^{5}$ The patient reported by Morse et al developed bilateral juxtapapillary choroidal neovascularisation and underwent laser photocoagulation in one eye when the choroidal neovascular membrane (CNV) grew towards the fovea.

The MPS (Macular Photocoagulation Study Group) showed that laser photocoagulation of well defined $\mathrm{CNV}$ in age related macular degeneration (AMD), presumed ocular histoplasmosis syndrome (POHS), and idiopathic $\mathrm{CNV}$, is beneficial..$^{6-10}$ In AMD, the treatment effect for subfoveal CNV is small, however, and some patients experience an immediate decline in central vision after laser treatment. Furthermore, many patients with AMD are ineligible for the treatment according to MPS criteria. ${ }^{11}$ Laser therapy has been used to treat CNVs caused by pseudotumour cerebri with limited success. ${ }^{12}$

Limitations in laser therapy for subfoveal $\mathrm{CNV}$, regardless of aetiology, have stimulated interest in alternative therapeutic modalities such as drug therapy with interferon alfa, ${ }^{12}$ teletherapy, ${ }^{13}$ and surgical excision of CNVs. ${ }^{14-17}$ Surgical excision of subfoveal CNVs in POHS can be associated with significant visual recovery. For example, Berger and Kaplan and Thomas et al reported 8/15 (53\%) and $6 / 16$ patients $(38 \%)$ with POHS improved two lines or more after CNV excision, respectively. ${ }^{14} 16$

In contrast, visual recovery following surgery for subfoveal $\mathrm{CNV}$ in patients with $\mathrm{AMD}$ is disappointing. ${ }^{15} 17$ Surgical excision of subfoveal CNV associated with other ocular conditions has also been reported by Adelberg et $a l^{18}$ including patients with angioid streaks, idiopathic CNV, and serpiginous choroiditis with variable outcomes. Differences in the duration of symptoms, in the anatomical location of the CNV (that is, subRPE in AMD), and in the regenerative capacity of senescent/ diseased RPE have all been adduced to explain why patients with POHS and idiopathic CNV seem to have better visual recovery than patients with AMD.

As far as we know, there have been no

reports of subfoveal CNV excision in patients with pseudotumour cerebri. The following is the clinical, angiographic, and histopathological correlation of the excised tissue in such a patient. 

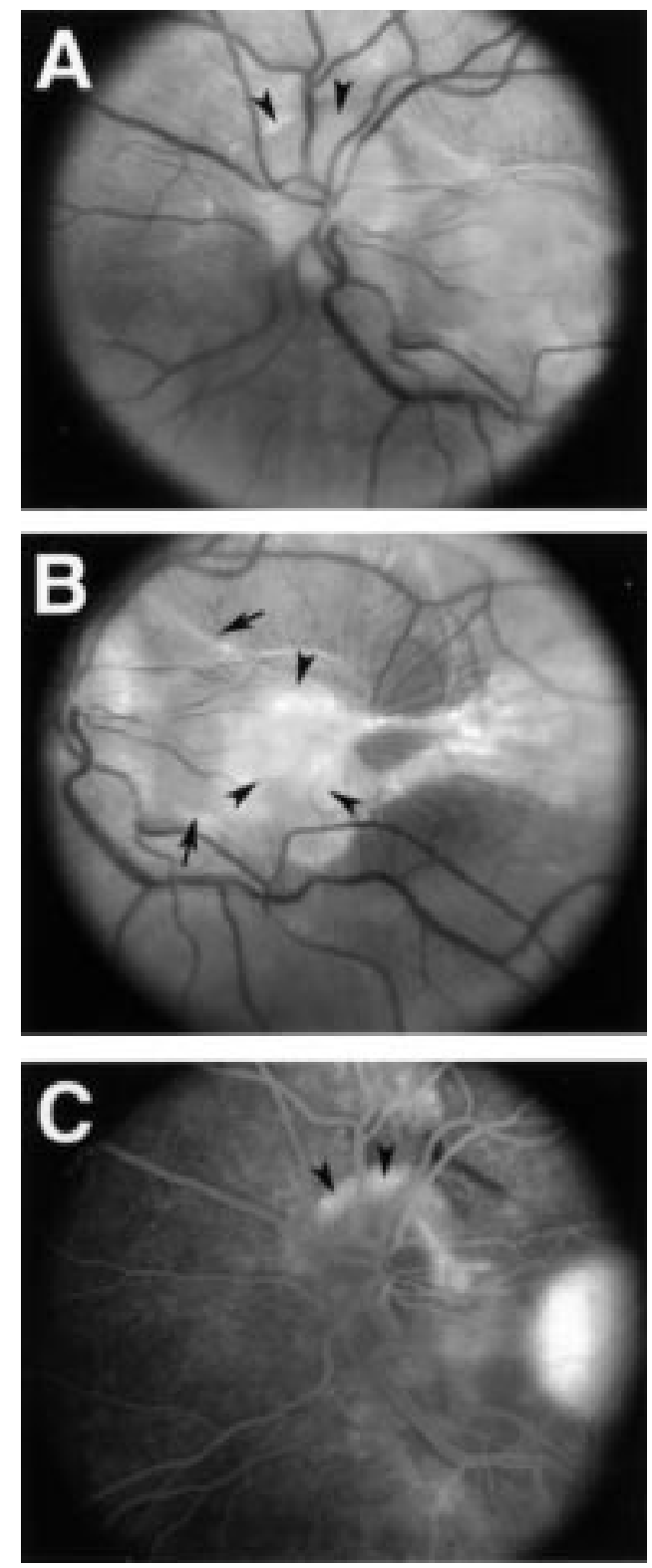

Figure 1 At 18 March 1988. (A) Fundus photograph discloses mild optic nerve swelling (arrowheads). (B) $A$ subfoveal choroidal neovascular membrane is present with a prominent white fibrous component (arrowheads) and surrounding choroidal folds (arrows). (C) Late frame of angiogram showing staining of the optic nerve head (arrowheads).

\section{Methods}

The patient underwent pre- and postoperative evaluation including measurement of best corrected visual acuity with Snellen and BaileyLovie charts and serial fluorescein angiography. Visual acuity was assessed by individuals experienced in low vision refraction and not part of the surgical team. Impaired choriocapillaris perfusion was judged to be present if choriocapillaris filling was delayed in the retinal arterial filling phase of the angiogram and persisted in the late phase of the study. CNV dimensions were recorded by measuring the largest horizontal and vertical dimensions of the CNV as determined with a reticle and the preoperative FA. The horizontal diameter of the optic disc was assumed to be $1500 \mu \mathrm{m}$.
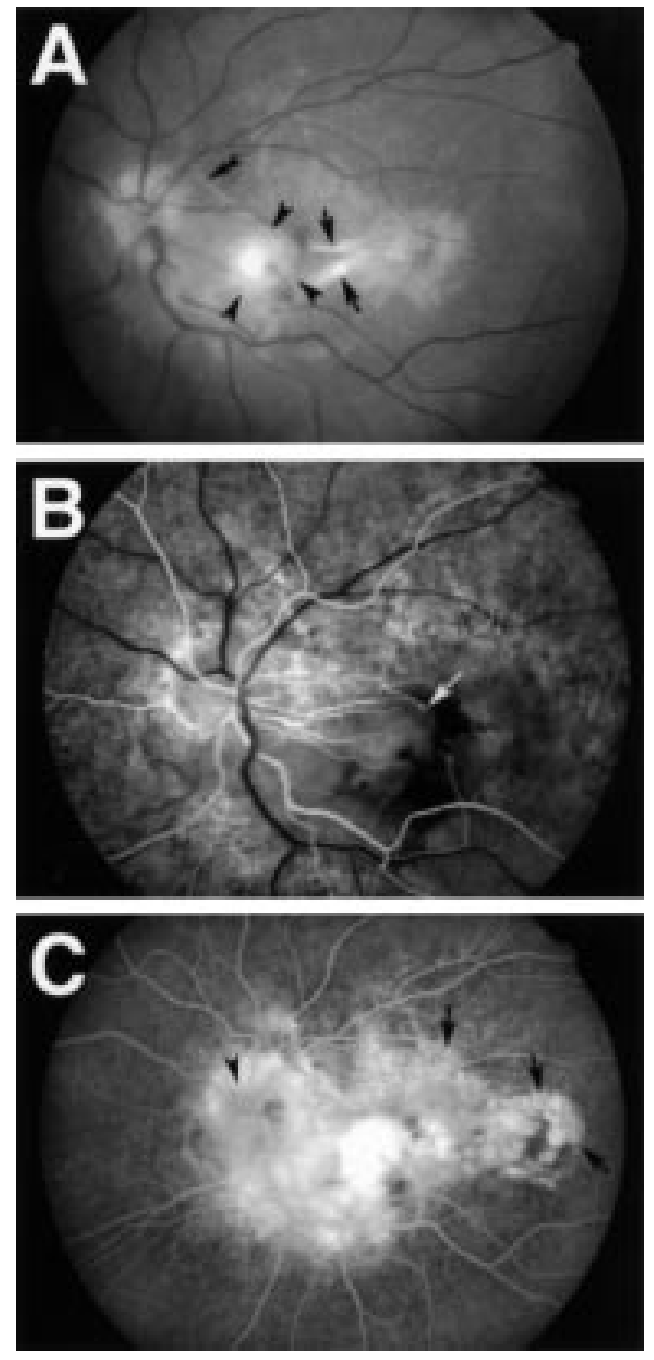

Figure 2 At 10 fune 1993. (VA =CF 6 inches) (A) The preoperative fundus photograph shows mild optic nerve pallor with mild papilloedema. There is a white subretinal scar (arrowheads) measuring approximately 1 disc diameter in size with a pigmented perimeter and a small amount of overlying haemorrhage. White fibrous strands

(arrows) extend to an area of RPE atrophy temporal to the fovea. A thinner fibrous band extends from the scar towards the optic nerve at the 1 o'clock position (double arrowhead). (B) The preoperative fluorescein angiogram shows early hyperfluorescence of the scar and the suggestion of a retinal-choroidal arterial anastomosis (arrow) at the temporal margin of the scar. There is no perfusion abnormality of the choriocapillaris in areas where it is not covered by subretinal fibrous tissue. (C) Late frame of the angiogram shown in (B) reveals window defects present temporally (arrows) which correspond to areas of $R P E$ atrophy or hypopigmentation. There is late staining of the optic nerve head (arrowhead).

The excised CNV was fixed initially in formol saline followed by immersion in half strength Karnowsky's fixative. The sample was then rinsed with several changes of phosphate buffered sucrose and postfixed in $2 \%$ osmium tetroxide. This step was followed by rinsing in the same buffered sucrose, dehydration in ethanol, and infiltration into Epon.

The tissue was polymerised for 2 days in a $60^{\circ} \mathrm{C}$ oven. Sections of $0.5 \mu \mathrm{m}$ were cut on a RMC MT-7 ultramicrotome and stained with toluidine blue for light microscopic evaluation. Thin sections were placed on large slot grids and were stained with uranyl acetate and lead 

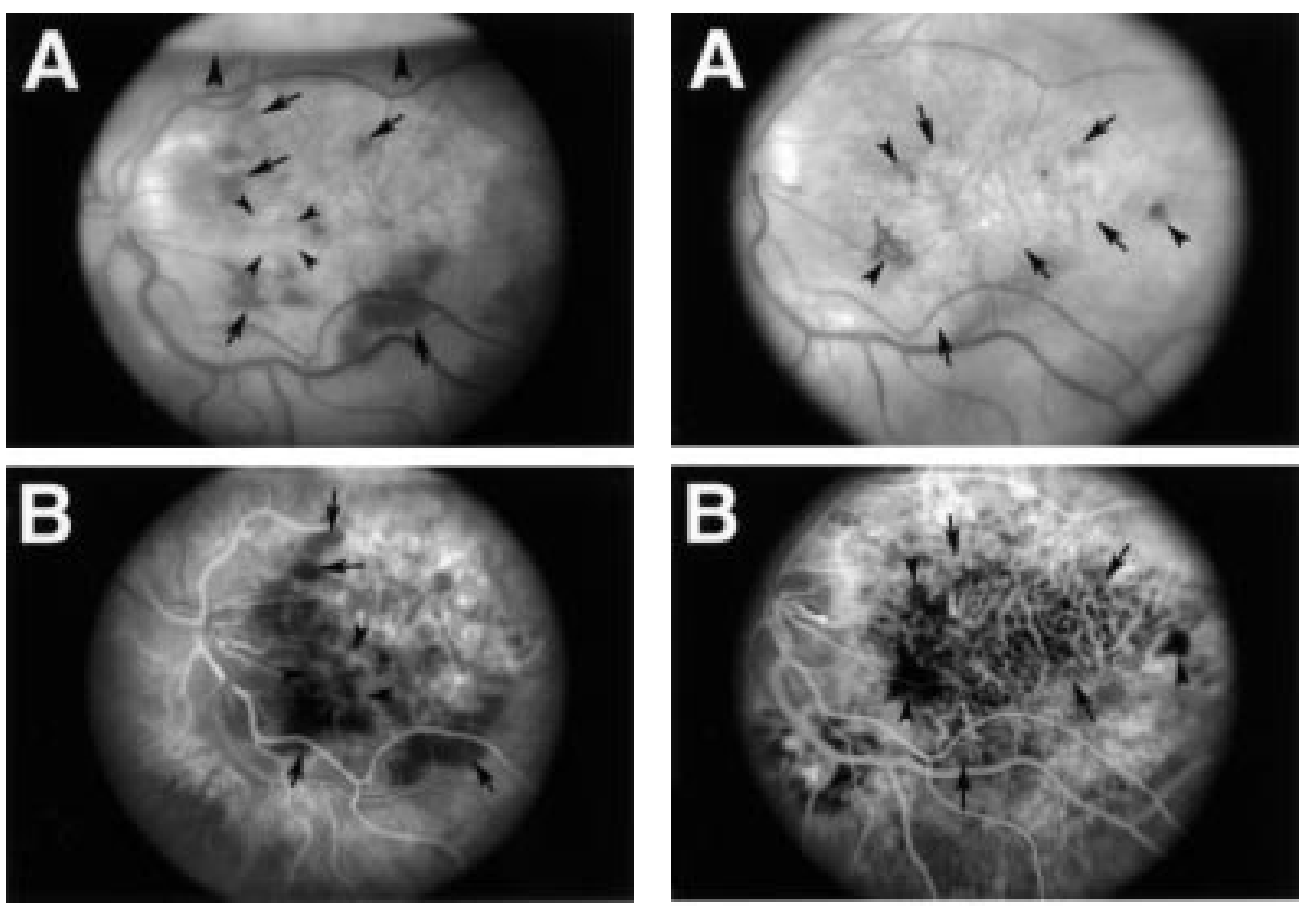

Figure 3 At 18 fune 1993. One week postoperatively the visual acuity was 1/200. (A) Fundus photograph and air-fluid meniscus (large arrowheads) is present just superior to the superotemporal arcade. Residual subretinal haemorrhage is visible (arrows). The previous location of the subretinal neovascular complex is outlined (small arrowheads). (B) Early venous filling phase fluorescein angiogram of $(A)$. Area of previous location of the neovascular complex shows mottled choriocapillaris filling (arrowheads). Areas with subretinal haemorrhage show blocked choroidal fluorescence (arrows).

citrate. Samples were examined and photographed on Zeiss EM 10 C electron microscope.

\section{Case report and results of angiography and histological studies}

A 32-year-old, obese white woman presented in February 1988 to her physician complaining of progressive visual loss in the left eye. The visual acuity was 20/20 right eye and 2/200 left eye, and a left relative afferent pupillary defect was present. The ophthalmologist noted a peripapillary subretinal fibrotic mass involving the left fovea that was thought to be due to a choroidal inflammatory lesion. Fundus photography and fluorescein angiography disclosed a subfoveal $\mathrm{CNV}$ associated with mild swelling and late staining of the optic nerve (Fig 1). The patient was treated with a posterior sub-Tenon prednisolone acetate (40 $\mathrm{mg}$ ) injection. There was no improvement.

Five years later, the patient was referred to the neuro-ophthalmology service at the University of California, San Francisco for evaluation of transient visual obscurations and progressive visual field loss in her right eye. Bilateral papilloedema due to pseudotumour cerebri was diagnosed based on the results of head computed tomography and lumbar puncture. The Snellen visual acuity was $20 / 20$ right eye and 2/200 left, and there was a left relative afferent pupillary defect. The fundus examination was significant for bilateral papilloedema with moderate optic nerve pallor in the left eye

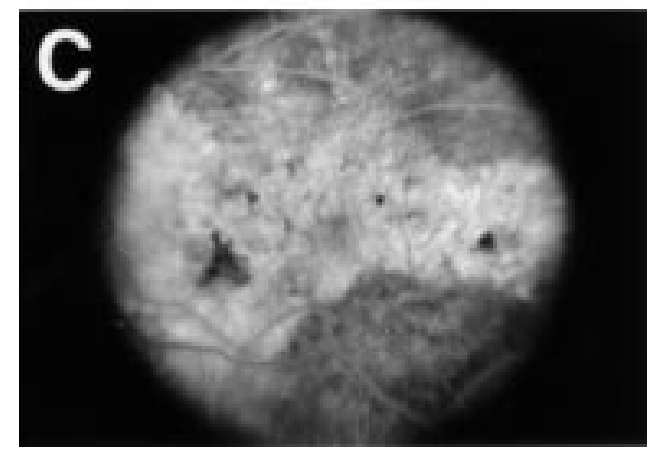

Figure 4 At 8 December 1993. Six months postoperatively the visual acuity was 2/200. (A) Fundus photograph shows new areas of visibility of the larger choroidal vessels (within the area outlined with arrows) compared with the preoperative appearance. Areas of RPE hyperplasia (arrowheads) are present along the border of the dissection bed. (B) Arterial phase angiogram of (A) shows marked choriocapillaris non-perfusion in the area outlined with arrows in $(A)$. The areas of RPE hyperplasia (arrowheads) block choroidal fluorescence. (C)

Recirculation phase of the angiogram shows filling of the large choroidal veins and persistent choriocapillaris non-perfusion.

(Fig 2A). In addition, a 1 disc diameter subretinal CNV with subfoveal extension and associated exudative retinal detachment was present in the left eye. The overlying retina showed prominent parafoveal cysts. The FA disclosed early hyperfluorescence of the scar with window defects temporally where there was RPE atrophy (Fig 2B, C). The patient underwent an uncomplicated left optic nerve sheath fenestration on 25 March 1993. Postoperatively, her visual symptoms resolved; the visual field stabilised, and the papilloedema decreased on the right. However, visual acuity in the left eye decreased mildly to counting fingers at 6 inches. The patient was offered the option of observation or surgical excision of the CNV. The patient elected to undergo CNV excision to stabilise the vision in the left eye 

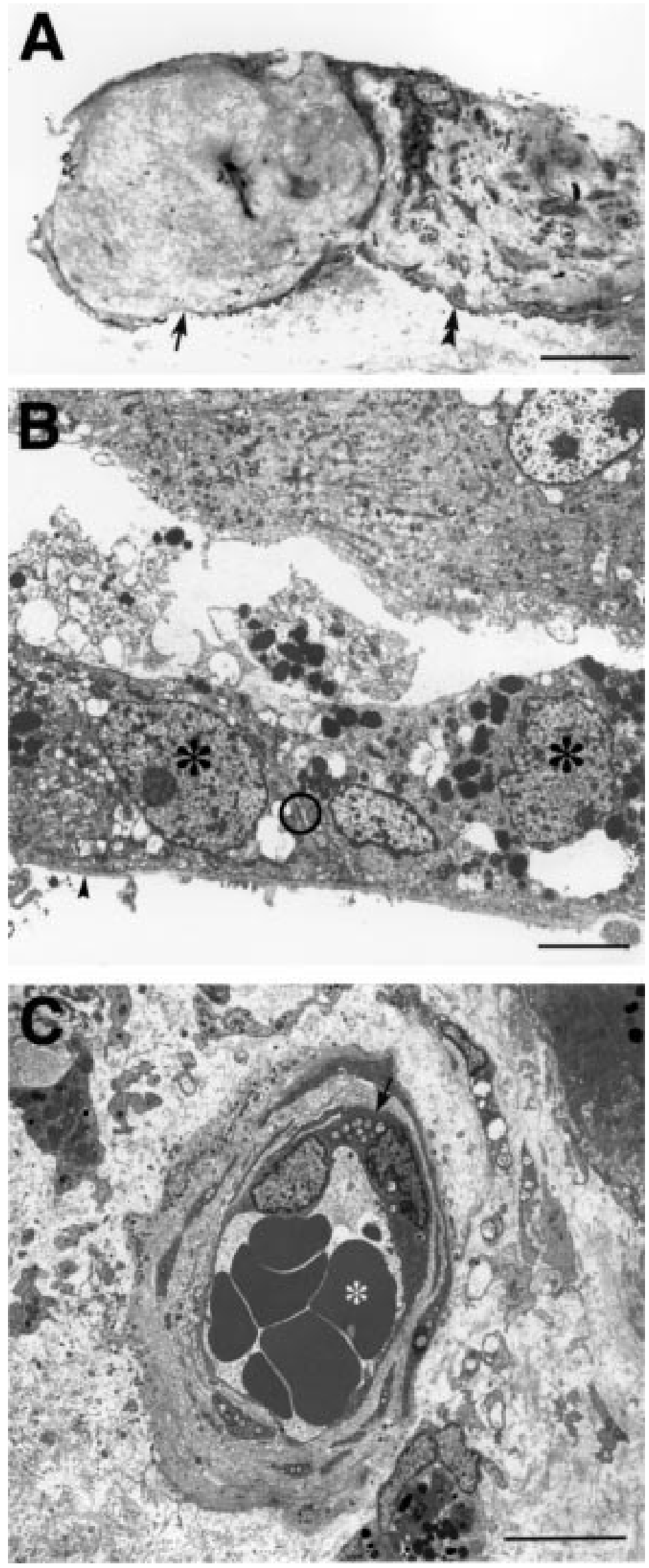

recognising that visual recovery would be limited by the chronicity of the disease.

A conventional three port vitrectomy was done. The posterior two thirds of the vitreous gel including the posterior vitreous cortex was excised. The preoperative FA was projected in the operating room to guide the subretinal dissection. There was a substantial exudative retinal detachment and prominent cystic change not just in the parafoveal area, but even outside the foveal avascular zone. The retina was puckered in an area of foveal adherence to the underlying scar. The subretinal fibrosis extended from the temporal margin of the optic nerve to the fovea (Fig 2A). Using a 33 gauge infusion cannula, the $\mathrm{CNV}$ was approached through an inferior retinotomy located just outside the inferotemporal arcade, approximately 2 disc diameters from the optic nerve. The CNV was freed from its underlying attachment to Bruch's membrane. Next, balanced salt was carefully infused to reveal the exact location of retina-CNV adhesions. Using gentle blunt dissection with a subretinal pick, the CNV was separated from its overlying attachment to the retina at the fovea and temporal to the optic nerve. A second retinotomy was made just inside the superotemporal arcade to dissect an adhesion along the superior-nasal surface of the scar which could not be reached through the initial retinotomy. Using blunt dissection, the adhesion between the scar and the retina was lysed, and the scar was completely freed from its overlying attachment to the retina without creating a retinal break. The Thomas forceps were introduced through the inferior retinotomy, and, with the intraocular pressure elevated, the CNV was grasped and gently pulled through the retinotomy, which enlarged to about three quarters of a disc diameter in size with a slit-like configuration conforming to the nerve fibre layer. A significant portion of the $\mathrm{CNV}$ appeared to lie beneath the RPE.

The vitreous cavity was washed out for 5 minutes to remove any liberated RPE. Vitreous incarceration was noted behind the superonasal sclerotomy, so prophylactic cryotherapy was applied posterior to each of the sclerotomy sites.

The retinotomies were not lasered, and a fluid-air exchange was performed flattening the retina completely.

The eye was then filled with a non-expansile concentration of $\mathrm{SF}_{6}$ gas, and the patient maintained a strict face down posture postoperatively until the gas bubble resorbed.

One week after surgery, an area of atrophy or hypopigmentation at the level of the RPE was evident in the previous location of the CNV

Figure 5 (A) Photomicrograph of excised CNV. The specimen is composed of a fibrous (arrow) and a vascular (double arrowhead) component. Magnification bar $=200$ $\mu m$. (B) Transmission electron micrograph of the internal surface of the specimen shows that it is lined by RPE cells (asterisks) joined by tight junctions (circle). Subjacent basement membrane (arrowhead) is present. Magnification bar $=5 \mu \mathrm{m}$. (C) Transmission electron micrograph from the centre of the specimen shows endothelial lined (arrow) vascular channels containing red blood cells (asterisk). Magnification bar $=5 \mu \mathrm{m}$. 

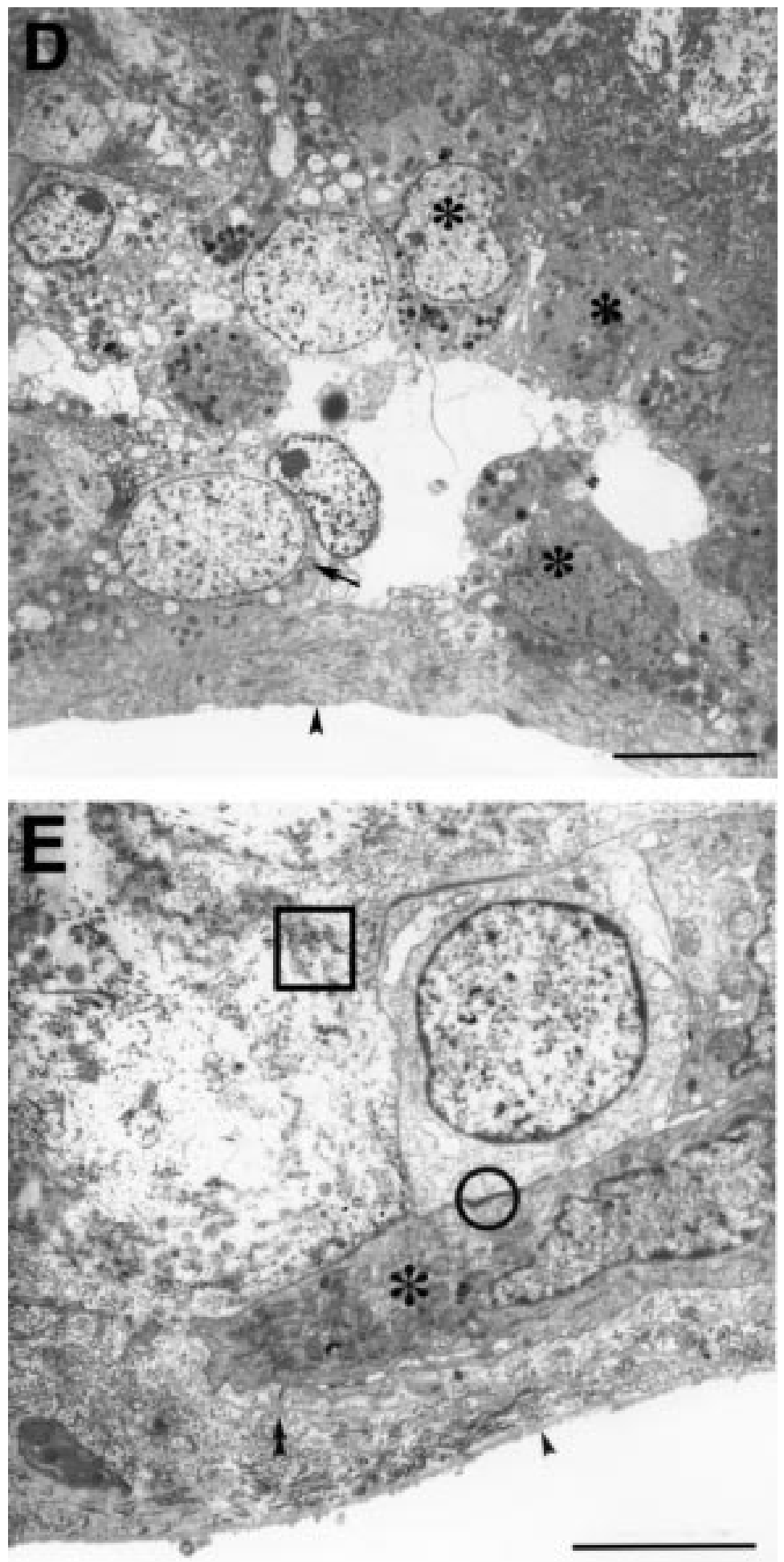

Figure 5 continued (D) Transmission electron micrograph of external surface of the specimen discloses presumed RPE basement membrane (arrowhead), an acinus of RPE cells (asterisks), and other unidentified cells (arrow). Magnification bar $=5 \mu \mathrm{m}$. (E) High magnification transmission electron micrograph of the external surface of the specimen discloses wide spaced collagen (square), presumed RPE basement membrane (arrowhead), flattened unidentified cells (possibly metaplastic RPE, asterisk) with reduplicated subjacent basement membrane (double arrowhead) and tight junctions (circle). Magnification bar = $5 \mu \mathrm{m}$.

(Fig 3A). Fluorescein angiography showed mottled subfoveal choriocapillaris fluorescence (Fig 3B). Six months after surgery visual acuity stabilised at $2 / 200$ in the left eye, and the retina remained flat (Fig 4A). Fluorescein angiography showed RPE atrophy associated with choriocapillaris non-perfusion in the area of the dissection bed (Fig 4B, C). Subsequently the patient's papilloedema increased in both eyes, and she began to develop optic pallor in the right eye. She has refused further interventions to lower her intracranial pressure.

Histologically, the excised CNV disclosed a specimen consisting of a vascular component and a collagenous scar (Fig 5A). Hyperplastic RPE lined the surface of the scar, and probable $\mathrm{RPE}$ were present in the interior of the scar. Electron microscopy showed discontinuous basement membrane lining the surface of the scar (Fig 5B, E). The vascular component of the $\mathrm{CNV}$ contained well defined vascular channels centrally (Fig 5C) and was surrounded by RPE (Fig 5A). Clumps of hyperplastic RPE, basement membrane (Figs 5D and $\mathrm{E}$ ), and wide spaced collagen (Fig 5E) were also present. Neither the collagenous components of Bruch's membrane nor the choriocapillaris were noted in the specimen.

\section{Discussion}

Idiopathic intracranial hypertension, commonly known as pseudotumour cerebri, is characterised by elevated cerebrospinal fluid (CSF) pressure, papilloedema, and normal CSF composition. ${ }^{19}$ Pseudotumour cerebri may be associated with identifiable aetiological factors such as middle ear disease, chronic obstructive pulmonary disease, radical neck dissection, non-specific infections, corticosteroid use or steroid withdrawal, or with other medications including vitamin A, tetracycline, and nalidixic acid. ${ }^{20}{ }^{21}$ Pseudotumour cerebri is also associated with obesity and pregnancy. ${ }^{21}$ In many cases the aetiology of the increased CSF pressure is unknown, and diminished CSF absorption and cerebral oedema have both been investigated as causes. ${ }^{22}{ }^{23}$

The major risk of a patient with pseudotumour cerebri is visual loss which occurs in $10 \%$ to $26 \%$ of patients. The primary cause of visual loss is progressive optic atrophy. Other causes include juxtapapillary choroidal neovascularisation, preretinal haemorrhage, central retinal vein occlusion, macular oedema (star), and chorioretinal folds. Our patient had both optic atrophy and choroidal neovascularisation with associated exudative retinal detachment and macular oedema as causes for visual loss in the left eye. The occurrence of juxtapapillary choroidal neovascularisation is a rare event, and, to the best of our knowledge, only five cases have been reported so far, two of them bilateral. ${ }^{1-5}$ There have been no reports of subfoveal CNV excision in the setting of pseudotumour cerebri. An unusual feature of this case is that the CNV was identified before the papilloedema was recognised clinically. However, bilateral fluorescein leakage from the optic nerve heads confirmed the presence of papilloedema at the time the CNV was noted in the left eye. The association of choroidal neovascularisation with papilloedema is not fully understood. Morse et $a l^{4}$ suggested that physical deformation of the peripapillary tissues by 
papilloedema may create the required pathway for the ingrowth of the neovascularisation beneath the retinal pigment epithelium and the sensory retina. Furthermore, hypoxia, created by axonal swelling induced impaired vascular perfusion, was suggested to contribute to the development of CNV.

In this patient, the CNV was located both external and internal to the RPE. The anatomical location of the $\mathrm{CNV}$ may affect visual outcome. ${ }^{24}$ If the $\mathrm{CNV}$ grows anterior to the RPE, excision may be accompanied by minimal removal of RPE-Bruch's membrane, but if the CNV grows posterior to the RPE, excision will create a localised RPE defect and may be associated with damage to subjacent Bruch's membrane.

In $\mathrm{AMD}$, the fibrovascular $\mathrm{CNV}$ may lie within Bruch's membrane, and both anterior and posterior to the RPE. CNV removal usually is associated with excision of RPE and portions of Bruch's membrane in such cases. In idiopathic and postinflammatory maculopathies, the CNV may grow anterior to the RPE into the subneurosensory subretinal space, but the CNV may also grow on both sides of the RPE. ${ }^{25}$ In our patient, a large RPE defect was created by the CNV excision. This defect was evident clinically and was corroborated by the presence of RPE in the excised tissue. Several observations in vivo and in vitro demonstrate the ability of the RPE surrounding a localised defect to repopulate the denuded surface of Bruch's membrane, although there is always some metaplasia and loss of pigmentation. ${ }^{26-29}$

The presence of mottled choriocapillaris fluorescence 1 week after surgery, and the development of choriocapillaris non-perfusion and RPE atrophy over the next 6 months raises the possibility that native RPE did not repopulate the dissection bed adequately. In previous reports involving AMD patients, we and others have described the occurrence of areas of decreased choriocapillaris perfusion in the previous location of the excised CNV. ${ }^{27} 3031$

In general, the presence of choriocapillaris non-perfusion after CNV excision might be due to pre-existing choriocapillaris atrophy masked preoperatively by the hyperfluorescence of the CNV on angiography; choriocapillaris damage/removal at the time of surgery; and RPE removal at surgery with abnormal RPE repopulation of the dissection bed and subsequent choriocapillaris atrophy. Based on the work of Korte et al in laboratory animals, ${ }^{32}$ the time course of the loss of choriocapillaris perfusion and the histology of the excised tissue suggest but do not prove that direct intraoperative trauma to the choroid was not the cause of these changes. Thus, we believe choriocapillaris non-perfusion in this case was secondary to abnormal/inadequate RPE repopulation of the dissection bed. In view of the patient's young age and the presumed normality of Bruch's membrane, we were surprised that choriocapillaris atrophy developed. However, the chronic exudative retinal detachment, which may have damaged the native RPE near the area of the dissection, and the size of the $\mathrm{CNV}$ and the corresponding RPE defect, may have played an important role in preventing adequate RPE resurfacing of the defect. The presence in the specimen of RPE cells associated with basement membrane may be important in this regard. The presence of Bruch's membrane has been correlated with higher rate of postoperative choriocapillaris atrophy following CNV excision. ${ }^{25}$

The authors have no proprietary interests in any of the instruments or materials mentioned in this study.

This study was supported in part by That Man May See, Inc, San Francisco, California, the New Jersey Lions Eye Research San Francisco, California, the New Jersey Lions Eye Research
Foundation, and Research to Prevent Blindness, Inc, New York (to M A Zarbin).

1 Jamison R. Subretinal neovascularization and papilledema associated with pseudotumor cerebri. Am f Ophthalmol 1978;85:78-81

2 Troost B, Sufit R, Grand M. Sudden monocular visual loss in pseudotumor cerebri. Arch Neurol 1979;63:440-2.

3 Morris A, Sanders M. Macular changes resulting from papilledema. Br f Ophthalmol 1980;64:211-6.

4 Morse PH, Leveille AS, Antel JP, Burch JV. Bilateral iuxtapapillary subretinal neovascularization associated with pseudotumor cerebri. Am f Ophthalmol 1981;91:312-7.

5 Caballero-Presencia A, Diaz-Guia E, Martinez-Perez M, Lopez-Lopez J. Nèo-vascularisation sous-rètinienne jiuxtapapillaire bilatèrale dans un cas de pseudotumor cerebri. $\mathcal{F}$ Fr Ophtalmol 1986;9:105-10.

6 Macular Photocoagulation Study Group. Argon laser photocoagulation for neovascular maculopathy. Three year results from randomized clinical trials. Arch Ophthalmol 1986;104:694-701.

7 Macular Photocoagulation Study Group. Krypton laser photocoagulation for neovascular lesions of ocular histophotocoagulation for neovascular lesions of ocular histothalmol 1987;105:1499-507.

8 Macular Photocoagulation Study Group. Krypton laser photocoagulation for idiopathic neovascular lesions. Results of a randomized clinical trial. Arch Ophthalmol 1990; 108:832-7.

9 Macular Photocoagulation Study Group. Laser photocoagulation of subfoveal neovascular lesions in age related macular degeneration. Updated findings from two clinical trials. Arch Ophthalmol 1993;111:1200.

10 Macular Photocoagulation Study Group. Visual outcome after laser photocoagulation for subfoveal choroidal neovascularization secondary to age related macular degeneration. Arch Ophthalmol 1994;112:480-8.

11 Freund KB, Yannuzzi LA, Sorenson JA. Age-related macular degeneration and choroidal neovascularization. $A m \mathcal{F}$ Ophthalmol 1993;115:786-91.

12 Thomas AM, Ibanez HE. Interferon alfa-2a in the treatment of subfoveal choroidal neovascularization. Am $\mathcal{f}$ Ophthalmol 1993;115:563-8.

13 Chakravarthy U, Houston RF, Archer DB. Treatment of age related subfoveal neovascular membranes by teletherapy:a pilot study. Br f Ophthalmol 1993;77:265.

14 Thomas MA, Kaplan HJ. Surgical removal of subfoveal neovascularization in the presumed ocular histoplasmosis syndrome. Am f Ophthalmol 1991;111:1-7.

15 Thomas MA, Dickinson JD, Melberg LS, Ibanez HE, Dhaliwal RS. Visual results after surgical removal of subfoveal choroidal neovascular membranes. Ophthalmology 1994;101:1384-96.

16 Berger AS, Kaplan HJ. Clinical experience with the surgical removal of subfoveal neovascular mebranes. Ophthalmology 1992;99:969-76.

17 Ormerod LD, Puklin JE, Frank RN. Long-term outcomes after the surgical removal of advanced subfoveal neovascular membranes in age related macular degeneration. Ophthalmology 1994;101:1201.

18 Adelberg D, Del Priore L, Kaplan H. Surgery for subfoveal membranes in myopia, angioid streaks, and other disormembranes in myopia, angioid

ders. Retina 1995; 15:198-205.
19 Miller N. Walsh and Hoyt's clinical neurophthalmology. Miller N. Walsh and Hoyt's clinical neurophthalm

20 Keltner JL, Miller NR, Gittinger JW. Pseudotumor cerebri. Surv Ophthalmol 1979;23:315-22.

21 Wall $M$, George D. Idiopathic intracranial hypertension. Brain 1991;114:155-80.

22 Boeri R. The pseudotumor cerebri. Curr Opin Neurol 1994; 7:69-73.

23 Karahalios DG, Rekate HL, Khayata MH, Apostolides PJ. Elevated intracranial venous pressure as a universal mechanism in pseudotumor cerebri of varying etiologies. Neurology 1996;46:198-202.

24 Gass JDM. Biomicroscopic and histopathologic considerations regarding feasibility of surgical excision of subfoveal neovascular membranes. Am f Ophthalmol 1994;118:285.

25 Grossniklaus HE, Hutchinson AK, Capone AJ, Woolfson J, Lambert MH. Clinicopathologic features of surgically excised choroidal neovascular membranes. Ophthalmology 1994;101:1099-111.

26 Heriot WJ, Machemer R. Pigment epithelial repair. Graefes Arch Clin Exp Ophthalmol 1992;230:91-100. 
27 Desai VN, Del Priore LV, Kaplan HJ. Choriocapillaris atrophy after submacular surgery in presumed ocular histoplas(19) ocular histop

28 Leonard DS, Zhang XG, Panozzo G, Sugino IK, Zarbin MA. Clinicopathological correlation of localized pigment epithelium debridement. Invest Ophthalmol Vis Sci 1997;38:1094-109.

29 Del Priore L, Hornbeck R, Kaplan H, Jones Z, Valentine TL, Mosinger-Ogilvie J, et al. Debridement of the pig retinal pigment epithelium in vivo. Arch Ophthalmol 1995;113 939-44.
30 Nasir MA, Sugino I, Zarbin MA. Decreased choriocapillaris perfusion following surgical excision of choroidal neovascular membranes in age-related macular degeneration. $\mathrm{BrF}$ Ophthalmol 1997;81:481-9.

31 Hudson HL, Frambach DA, Lopez PF. Relation of the functional and structural fundus changes after submacular surgery for neovascular age-related macular degeneration. Br f Ophthalmol 1995;79:417-23.

32 Korte GE. Choriocapillaris regeneration in the rabbit. Invest Ophthalmol Vis Sci 1989;30:1938-50. 